\title{
A new gradient method via least change secant update
}

\begin{abstract}
The Barzilai-Borwein (BB) gradient method is favourable over the classical steepest descent method both in theory and in real computations. This method takes a 'fixed' step size rather than following a set of line search rules to ensure convergence. Along this line, we present a new approach for the two-point approximation to the quasi-Newton equation within the BB framework on the basis of a well-known least change result for the Davidon-Fletcher-Powell update and propose a new gradient method that belongs to the same class of BB gradient method in which the line search procedure is replaced by a fixed step size. Some preliminary numerical results suggest that improvements have been achieved.
\end{abstract}

Keyword: Gradient methods; Barzilai-Borwein method; Conjugate gradient method; QuasiNewton equation; Least change update 